\title{
Índice Glucémico in vitro, Contenido fenólico y Actividad Antioxidante de Snacks Elaborados con Harinas de Plátano (Musa paradisiaca) y Yacón (Smallanthus sonchifolius)
}

\begin{abstract}
Alejandra Díaz-Osorio(1), Marcela Martínez-Castaño(2), José Contreras-Calderon ${ }^{(1)}$ y Cecilia Gallardo-Cabrera ${ }^{(2) \star ~}$ (1) Universidad de Antioquia, Facultad de Ciencias Farmacéuticas y Alimentarias, Grupo de investigación BIOALI, Cl. Barranquilla \#53 - 108, Medellín-Colombia. (e-mail: alejandra.diazo@udea.edu.co, jose.contrerasc@udea.edu.co) (2) Universidad de Antioquia, Facultad de Ciencias Farmacéuticas y Alimentarias, Grupo de investigación GEMCA, Cl. Barranquilla \#53 - 108, Medellín-Colombia. (e-mail: marcela.martinez1@udea.edu.co; cecilia.gallardo@udea.edu.co)
\end{abstract}

* Autor a quien debe de ser dirigida la correspondencia

Recibido Dic. 13, 2018; Aceptado Feb. 25, 2019; Versión final Mar. 26, 2019, Publicado Oct. 2019

\begin{abstract}
Resumen
El objetivo del estudio fue desarrollar un snack de harina de plátano y yacón aprovechando su potencial en la formulación de alimentos saludables. Se prepararon cuatro formulaciones de snacks a base de harina de plátano con adición de harina de yacón $(0,7,13$ y $20 \%$ ). La harina de yacón favoreció el aumento de tonalidades amarillas y rojizas, contenido de polifenoles totales y la actividad antioxidante en las formulaciones. Todas las formulaciones mostraron menor área en la curva de hidrólisis del almidón respecto al pan blanco. El snack con $13 \%$ de harina de yacón obtuvo mayor preferencia sensorial, con un contenido de fenoles totales (3.93 mg Equivalente Ácido Gálico / g), actividad antioxidante (4.99 $\mu \mathrm{M}$ Equivalente Trolox / g) y un bajo índice glucémico estimado (51.04). Basado en los resultados del estudio, se concluye que estos snacks podrían ser producidos industrialmente, constituyendo una buena fuente alternativa en antioxidantes y en carbohidratos no digeribles.
\end{abstract}

Palabras clave: alimento funcional; harina; índice glucémico; acción antioxidante

\section{Glycemic Index in vitro, Phenolic Content and Antioxidant Activity of a Snacks made with unripe banana (Musa paradisiaca) and Yacon (Smallanthus sonchifolius) Flours}

\begin{abstract}
The objective of the study was to develop a snack from unripe banana and yacon flours taking advantage of their potential in the formulation of healthy foods. Four formulations of snacks based on unripe banana flour with addition of yacon flour (0, 7, 13 and 20\%) were prepared. Yacon flour favored the increase of yellow and reddish tones, total polyphenol content and antioxidant activity in the formulations. All formulations showed lower area in the hydrolysis curve of the starch with respect to that of white bread. Snack with $13 \%$ yacon flour obtained greater sensory preference, with a total phenol content $(3.93 \mathrm{mg}$ Gallic Acid Equivalent $/ \mathrm{g}$ ), antioxidant activity (4.99 $\mu \mathrm{M}$ Equivalent Trolox / g) and a low estimated glycemic index (51.04). Based on the results of the study, it is concluded that these snacks could be industrially produced, constituting a good alternative source of antioxidants and non-digestible carbohydrates.
\end{abstract}

Keywords: functional food; flour; glycemic index; antioxidants 


\section{INTRODUCCIÓN}

Los cambios en el estilo de vida debido a la urbanización de la población han conllevado al aumento en la incidencia de enfermedades no transmisibles como la obesidad y la diabetes, donde ciertos patrones de dieta son uno de los factores que aumentan el riesgo de contraer estas enfermedades, y cuyos mecanismos biológicos aún no están completamente determinados. Los carbohidratos son la fuente de energía más importante para la mayoría de las culturas, por eso diversos estudios se han enfocado en evaluar la influencia de la cantidad y calidad de los carbohidratos consumidos en la manifestación de estas enfermedades. Las evidencias sugieren que la elevación frecuente de la glucemia postprandial es uno de los factores de riesgo para la diabetes y sus complicaciones patológicas asociadas (García Soidán, 2003); como también para la obesidad y enfermedades cardiovasculares en pacientes no diabéticos (Augustin et al., 2015). En 1980, se introdujo el concepto de índice glucémico, IG, para medir el impacto de un alimento en la glucemia postprandial, con una escala de 0-100 y clasificando los alimentos como productos con IG: alto (>70), intermedio (55-70) y bajo (<55). El IG se puede estimar siguiendo métodos in vitro, denotándose como elG para diferenciarse de aquel obtenido por métodos in vivo El IG está determinado por la velocidad de digestión y absorción de los carbohidratos a nivel gastrointestinal. Carbohidratos con una rápida absorción dan lugar a altos valores de IG, mientras que aquellos de lenta absorción producen respuestas glucémicas más planas y en consecuencia bajos IG (Ludwig, 2002). Factores adicionales a la naturaleza química del carbohidrato, como la microestructura del alimento, proceso tecnológico, entre otros, pueden alterar su biodisponibilidad (Parada y Rozowski, 2008). El almidón se clasifica en tres clases según la velocidad de digestión y absorción: almidón rápidamente digerible (ARD), almidón lentamente digerible (ALD) y almidón resistente (AR). El conocimiento y manejo adecuado de los factores que afectan la biodisponibilidad del almidón abre un nuevo campo de investigación, y plantea la formulación de alimentos con modulación de la respuesta glucémica que cubran las necesidades dietarías de pacientes diabéticos o con sobrepeso (Magallanes-Cruz et al., 2017).

El uso de plátano verde, como ingrediente funcional ha atraído mucha atención debido a su alto contenido de almidón resistente (García-Solís et al., 2018), su efecto hipoglucémico reconocido tradicionalmente (Shodehinde et al., 2015), y su potencial nutricional y nutracéutico como fuente de compuestos antioxidantes (Agama-Acevedo et al., 2012). Por otra parte, las propiedades sensoriales del plátano son relevantes, siendo los snacks de plátano frito uno de los productos más vendidos en países tropicales; despertando el interés tecnológico para mejorar su perfil nutricional, mediante la implementación de nuevas tecnologías de proceso para disminuir el contenido graso, grado de rancidez (Chavez-Salazar et al, 2017; Ikegwu, O. J et al, 2018) y formación de acrilamida (Shamla y Nisha, 2017). A pesar del conocimiento de las bondades del plátano, solo escasas propuestas se han hecho sobre su aprovechamiento en la formulación de snacks con bajo índice glucémico (Utrilla-Coello et al., 2011; Flores-Silva et al., 2015). Los snacks son productos que buscan satisfacer la saciedad entre comidas, que se caracterizan en general por un alto contenido de carbohidratos. Dado las nuevas tendencias de los consumidores, el desarrollo de snacks con beneficio en la salud es una oportunidad del sector alimentario (Van Kleef et al., 2012). Otro material particular, en el contexto de productos con carbohidratos de respuesta glucémica interesante es el yacón (Smallanthus sonchifolius) debido a su alto contenido de fructooligosacáridos (FOS), del 50 - 70\% de los carbohidratos totales (Valdez Clinis et al., 2013). Los FOS son un tipo de fibra tipo fructano, oligosacáridos lineales de fructosa, que no son hidrolizados por las enzimas digestivas, sino que son fermentados por la flora intestinal, actuando como prebióticos al estimular el crecimiento de bacterias benéficas en el intestino delgado (Sousa et al., 2015). Además, el yacón también es reconocido por su alto contenido de polifenoles (Khajehei et al., 2018). Por lo anterior el yacón posee un alto potencial para ser usado tanto en productos farmacéuticos como alimentarios. Sin embargo, presenta dos desventajas, su alto contenido acuoso $(>80 \%)$ y la alta actividad de polifenoloxidasas, que lo convierte en un producto altamente perecedero y con dificultades para conservar sus propiedades durante el proceso de secado y almacenamiento, lo que ha limitado sus aplicaciones (Castro et al., 2012).

Uno de los métodos de secado que se ha estado usando en otras matrices alimentarias para contrarrestar las dificultades, es el microondas con vacío, cuya combinación permite por una parte disminuir la presión de vapor del líquido, de modo que la deshidratación de los productos sensibles se puede realizar en ausencia de oxígeno y la radiación de las microondas se transforma en energía cinética en el producto aumentando la eficiencia y uniformidad en el secado (Schulze et al., 2014). La radiación de microondas, también, se está usando para hornear productos finales debido al bajo el tiempo de exposición al calor que reduce el avance de procesos de descomposición de biomoléculas entre ellos la hidrólisis del almidón (Allen et al., 2012). Por lo anterior y dado que la combinación de la harina de plátano y yacón no ha sido estudiada en nuestro conocimiento, el presente trabajo tuvo como objetivo desarrollar un alimento tipo snack a base de harina de plátano fortificado con harina de yacón, evaluando el efecto en la actividad antioxidante, color, índice glucémico estimado por método in vitro (e/G) y digestibilidad del almidón, así como en sus propiedades sensoriales. 


\section{METODOLOGÍA}

En el desarrollo experimental se utilizó harina de plátano verde (HPL), que se adquirió en un mercado local de la ciudad de Medellín (Colombia). La harina de yacón (HYC) fue elaborada a partir de tubérculos de yacón (Smallanthus sonchifolius) variedad amarillo, adquiridos de un mismo cultivo ubicado en el municipio de Honda (Tolima-Colombia) y procesados al segundo día de la cosecha.

\section{Obtención de la harina de yacón}

El yacón fue pelado y cortado en rodajas de $4 \mathrm{~mm}$ de espesor. Luego las rodajas fueron secadas en microondas con vacío de escala semi-industrial con magnetrones (CW $2450 \mathrm{MHz}$, Toshiba, China) y bomba de vacío de paletas rotatorias (Lanphan $2 X Z-4$, Zhengzhou, China) operado a una presión de vacío de -80 $\mathrm{KPa}$, a una temperatura de $50^{\circ} \mathrm{C}$, potencia de entrada $(1.6 \mathrm{KW})$, potencia de salida $(0.3 \mathrm{KW})$ y tiempo de secado de $1.2 \mathrm{~h}$. Finalmente, el producto seco se molió en molino analítico IKA® A1 con un tamaño de partícula de $150 \mu \mathrm{M}$ para obtener la harina, que luego fue empacada al vacío y almacenada a temperatura ambiente para los posteriores análisis.

\section{Formulación de snacks}

Se realizaron cuatro formulaciones variando el contenido de HYC y manteniendo constante la HPT, el contenido de agua y sal, en las cantidades descritas en la Tabla 1. Las materias primas fueron mezcladas manualmente hasta integrar todos los ingredientes, posteriormente se extendió la masa a un espesor de 3 $\mathrm{mm}$ y se cortó con un molde circular de $5 \mathrm{~cm}$ de diámetro. Los snacks se hornearon en un microondas durante $80 \mathrm{~s}$, en un microondas comercial (HACEB) con potencia de salida de 1000W, hasta un contenido de humedad de $10 \pm 2 \%$.

Tabla 1: Diseño de formulación de snacks de harina de plátano (HPT) con adición de harina de yacón (HYC)

Análisis de color

\begin{tabular}{|l|c|c|c|c|}
\hline \multirow{2}{*}{ Ingrediente } & \multicolumn{4}{|c|}{ Código de la formulación } \\
\cline { 2 - 5 } & $Y 0$ & $Y 7$ & $Y 13$ & Y20 \\
\hline HPT, g & 100 & 100 & 100 & 100 \\
\hline HYC, g/100 g HPT & 0 & 7 & 13 & 20 \\
\hline Sal, g/100 g HPT & 3 & 3 & 3 & 3 \\
\hline Agua, g/100 g HPT & 85 & 85 & 85 & 85 \\
\hline
\end{tabular}

El color fue determinado con un espectro colorímetro (ColorFlex EZ - HunterLab) con referencia del iluminante D65 y observador de $10^{\circ}$ para medir las coordenadas del sistema CIELAB: $L^{*}$ (luminosidad), $a^{*}$ (+a rojo y -a verde) y $b^{*}(+b$ amarillo y $-b$ azul), cada muestra se analizó por triplicado. Además, con estas coordenadas se calcularon el tono (h) y croma (C) mediante las ecuaciones 1 y 2 (Alvis-Bermudez et al., 2016):

$$
\begin{aligned}
& \mathrm{h}=\operatorname{arctg}\left(\left(\mathrm{b}^{\star}\right) /\left(\mathrm{a}^{\star}\right)\right) \\
& \mathrm{C}^{*}=\sqrt{ }\left(\mathrm{a}^{* \wedge} 2+\mathrm{b}^{* \wedge} 2\right)
\end{aligned}
$$

\section{Preparación de la muestra para determinación de capacidad antioxidante y polifenoles totales}

La extracción de la muestra para la determinación de los polifenoles totales y la actividad antioxidante de los snacks se realizó de acuerdo a Contreras-Calderón et al. (2016). $0.5 \mathrm{~g}$ de muestra fueron pesados en tubos falcón de $10 \mathrm{~mL}$ junto con $8 \mathrm{~mL}$ de una mezcla metanol: agua (50:50, $\mathrm{pH}$ 2), luego se agitó durante $1 \mathrm{~h}$ a temperatura ambiente. Posteriormente, se centrifugaron los tubos a $5000 \mathrm{rpm}$ durante $10 \mathrm{~min}$, se filtró y se recogió el filtrado en un balón volumétrico de $25 \mathrm{~mL}$. El residuo, se extrajo nuevamente con $8 \mathrm{~mL}$ de una mezcla acetona: agua (70:30) y se repitió el proceso nuevamente. Los filtrados se mezclaron y aforaron con agua destilada. Los extractos obtenidos se almacenaron en envases ámbar a $-18^{\circ} \mathrm{C}$ hasta su análisis. Los extractos se realizaron por triplicado.

\section{Contenido de polifenoles totales, CPT}

El contenido fenólico total se determinó utilizando el Ensayo Folin- Ciocalteau según la metodología planteada por (Contreras-Calderón et al., 2016), con algunas modificaciones. Brevemente, $20 \mu \mathrm{L}$ del extracto se mezclaron con $1580 \mu \mathrm{L}$ de agua desionizada, $100 \mu \mathrm{L}$ de reactivo Folin-Ciocalteu (Applichem-PanReac) y 300 $\mu \mathrm{L}$ de solución de carbonato de sodio al $20 \%$. La mezcla se agitó y se mantuvo a temperatura ambiente y en 
oscuridad durante $60 \mathrm{~min}$. Seguidamente se midió absorbancia a $725 \mathrm{~nm}$ en un espectrofotómetro (Thermo Scientific, Evolution 60S). Soluciones acuosas de ácido gálico (entre 0 y $1000 \mathrm{ppm}$ ) se usaron para la curva de calibración. Los resultados se expresaron como mg de equivalentes de ácido gálico (EAG) por g de muestra (mg de EAG/g de muestra).

\section{Actividad antioxidante, ABTS}

El ensayo ABTS se realizó siguiendo la metodología descrita por Contreras-Calderón et al. (2011). Brevemente, $100 \mu \mathrm{L}$ del extracto se mezcló con $1000 \mu \mathrm{L}$ de solución del radical ABTS + y se incubó a $30^{\circ} \mathrm{C}$ por $30 \mathrm{~min}$. La curva de calibración fue realizada con soluciones acuosas de Trolox en concentraciones entre 0 y $250 \mu \mathrm{mol}$. Las absorbancias se midieron a $730 \mathrm{~nm}$ en un espectrofotómetro (Thermo Scientific, Evolution $60 S$ ). Los resultados se expresaron como micro moles de equivalentes de Trolox por gramo de muestra ( $\mu \mathrm{mol}$ de Trolox /g).

\section{Digestibilidad in vitro del almidón e índice glucémico estimado}

Un método in vitro modificado basado en el procedimiento de Goñi et al. (1997) fue adoptado para determinar la digestibilidad in vitro del almidón para los snacks formulados. La muestra molida $(50 \mathrm{mg})$ se dispersó en 10 $\mathrm{mL}$ de tampón $\mathrm{HCl}-\mathrm{KCl}(\mathrm{pH}$ 1.5) y se adicionó $200 \mu \mathrm{L}$ de pepsina (Sigma-Aldrich; $125 \mathrm{U} / \mathrm{mL}$ ), luego se agitó durante $1 \mathrm{~h}$ en un baño de agua a $37^{\circ} \mathrm{C}$. Posteriormente, el pH se ajustó a 6.9 usando tampón tris-maleato (pH 6.9) y ajustó a un volumen de $25 \mathrm{~mL}$. Se añadieron $52 \mu \mathrm{L}$ de $\alpha$-amilasa (Sigma-Aldrich; $50 \mathrm{U} / \mathrm{mL}$ ) y se incubó a $37^{\circ} \mathrm{C}$ en un baño de agua con agitación. Se extrajeron alícuotas de $1 \mathrm{~mL}$ de muestra en diferentes tiempos (30, 90,120 ,180 y $200 \mathrm{~min}$ ); cada una se inactivó colocando inmediatamente el tubo en un baño de agua hirviendo durante $5 \mathrm{~min}$. Las alícuotas se almacenaron a temperaturas de refrigeración $(4 \stackrel{\circ}{\circ}$ ) hasta obtener la última alícuota. A todas las alícuotas se les añadió $3 \mathrm{~mL}$ de tampón de acetato sódico $0,4 \mathrm{M}(\mathrm{pH}$ 4,75 ) y $200 \mu \mathrm{L}$ de amiloglucosidasa (Xi'an Lyphar Biotech Co., LTD; $330 \mathrm{U} / \mathrm{mL}$ ) y se incubaron a $60^{\circ} \mathrm{C}$ durante 5 min para hidrolizar el almidón a glucosa. Una alícuota de $100 \mu \mathrm{L}$ fue incubada con glucosa oxidasa / peroxidasa (GOD/POD) (Biosystems S.A, España). La absorbancia se midió a $510 \mathrm{~nm}$ contra el blanco de reactivo y usando como estándar una solución de glucosa, en un espectrofotómetro (Thermo Scientific, Evolution 60S). El contenido de almidón se calculó multiplicando la glucosa (mg) por un factor de 0.9 .

Para la determinación del porcentaje de fracciones de almidón, se denominó ARD a la fracción de almidón hidrolizado dentro de los 30 min de incubación, ALD a la fracción hidrolizada entre 30 y 180 min y AR a la fracción que se hidrolizó entre 180 y 200min. El almidón total, AT, se calculó como la suma de las fracciones. El índice de hidrólisis, IH, y el índice glucémico estimado (elG) de los snacks y el pan blanco como control, se calcularon a partir de la ecuación (3) que corresponde a una cinética de primer orden de la hidrólisis del almidón.

$$
\left[C=C_{\infty}(1-e-k t)\right]
$$

Donde $\mathrm{C}=$ concentración de almidón en cada momento, $\mathrm{C} \infty=$ concentración del almidón de equilibrio de la hidrólisis y $\mathrm{k}=$ constante de velocidad. El IH se calculó como la relación entre el área bajo la curva de hidrólisis (0-200min) de muestras de snacks respecto a la del pan blanco; luego se calculó elG utilizando la ecuación (4) propuesta por (Grandfeldt et al., 1992)

$\mathrm{elG}=8.198+0.862 \mathrm{HI}$

\section{Análisis sensorial}

Se realizó una prueba sensorial con evaluadores seleccionados y semi-entrenados, conformado por 30 personas, a las cuales se les solicitó evaluar la aceptación general de las formulaciones fortificadas con HYC $(7,13$ y $20 \%)$. La prueba usada fue de ordenamiento preferencial hedónica según los parámetros de la ISO 8587, (2006).

\section{Análisis estadístico}

Se realizó un análisis de varianza (ANOVA) con un nivel de significancia del 95\% para determinar las diferencias entre los parámetros de color, contenido de polifenoles totales, actividad antioxidante, digestibilidad del almidón e índice glicémico in vitro a través del software Statgraphics Centurion XVII versión. Para el análisis estadístico de los resultados de la prueba sensorial se recurrió a la prueba de Friedman con un nivel de significancia del $95 \%$ y prueba de Fischer con un nivel de confianza del $99 \%$, donde la hipótesis nula $\mathrm{H}_{0}=$ la suma de los rangos de las muestras no presenta diferencias y la hipótesis alternativa $\mathrm{H}_{\mathrm{a}=}$ la suma de los rangos de las muestras presenta diferencias. Si $\mathrm{F}_{\text {Prueba }}>\mathrm{F}_{\mathrm{Valor}}$ Crítico la hipótesis nula se rechaza. 


\section{RESULTADOS Y DISCUSIÓN}

Los resultados se presentan en cinco secciones: i) Evaluación de las harinas, ii) Análisis colorimétrico de los snacks iii) Análisis de polifenoles totales y actividad antioxidantes ABTS de los snacks, iv) Análisis del índice glucémico estimado y digestibilidad del almidón de los snacks y v) Análisis sensorial de los snacks.

\section{Evaluación de las harinas}

El análisis de color es un factor importante en la calidad de las harinas, debido a que indica la conservación o descomposición de los cromóforos o pigmentos durante los procesos de obtención. El análisis de las coordenadas de color CIELab y CIELCh (Tabla 2) indicó que la HPT es más blanca que la HYC, tal como se esperaba de acuerdo con la naturaleza del material de partida. La HYC presentó un mayor valor en el componente amarillo del color $\left(b^{*}\right)$ y con mayor intensidad $\left(C^{*}\right)$, estos valores indicaron la persistencia de los pigmentos en la harina. Se ha reportado que el yacón durante la deshidratación sufre oscurecimiento debido a la oxidación enzimática de compuestos fenólicos por la alta actividad de las enzimas peroxidasas y polifenoloxidasas, que se evidencia en la disminución de la luminosidad L (Benítez-Franco et al., 2016). Con el método de secado por microondas con vacío se obtuvo un producto con un color amarillo puro, tal como se muestra en la Figura 1, además la harina obtenida mostró mayor índice de luminosidad $L^{*}$ y menor valor de la coordenada $\mathrm{a}^{*}$ que esos obtenidos en secado por convección a temperaturas entre $45-60{ }^{\circ} \mathrm{C}$ (Salinas et al., 2018). El incremento en la coordenada $\mathrm{a}^{*}$ pueden estar relacionado con la tendencia al pardeamiento no enzimático. En la Tabla 2 los resultados se expresan como la media \pm desviación estándar media, $(n=3)$.

Tabla 2: Parámetros colorimétricos, contenido de fenoles (CPT) y actividad antioxidante ABTS de las harinas de plátano (HPT) y de yacón (HYC).

\begin{tabular}{|c|c|c|c|c|c|c|c|}
\hline Harina & $L^{*}$ & $a^{*}$ & $b^{*}$ & $C^{*}$ & $h^{*}$ & $\begin{array}{c}C P T \\
{[\mathrm{mg} E A G / \mathrm{g}]}\end{array}$ & $\begin{array}{c}A B T S \\
{[\mu m o l E T / g]}\end{array}$ \\
\hline HPT & $82.00 \pm 0.09$ & $0.70 \pm 0.02$ & $14.02 \pm 0.07$ & $14.04 \pm 0.07$ & $87.13 \pm 0.00$ & $5.93 \pm 0.43$ & $13.96 \pm 0.04$ \\
\hline HYC & $64.99 \pm 0.60$ & $5.10 \pm 0.24$ & $30.51 \pm 0.42$ & $30.94 \pm 0.45$ & $80.45 \pm 0.05$ & $31.06 \pm 0.67$ & $87.13 \pm 10.64$ \\
\hline
\end{tabular}
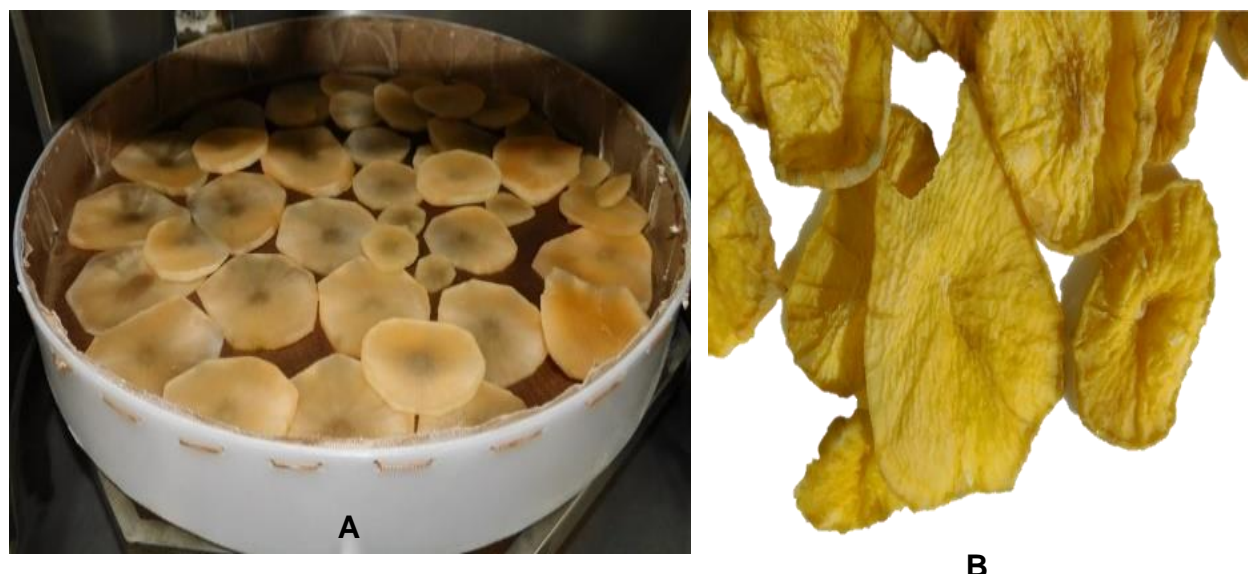

Fig. 1: Fotografías del proceso de secado de yacón usando microondas con vacío. A: Yacón antes de secar, B: Yacón deshidratado

Respecto al CPT, se observó mayor contenido en la HYC que en la HPT (Tabla 2). El CPT encontrado en la HYC del presente estudio fue superior al valor reportado por Salinas et al. (2018) (10.1 mg EAG / g); y Castro et al. (2012) (18.6 mg EAG / g) indicando que el proceso de microondas con vacío disminuye la conversión de los fenoles en quinonas por la acción de las polifenoloxidasas (Queiroz et al., 2011). El CPT encontrado en HPT estuvo dentro del rango reportado para diferentes tipos de cultivo de plátano, 1 - $11 \mathrm{mg} \mathrm{EAG/g}$ (Anyasi, Jideani y Mchau, 2018) y 2.32 - 9.9 mg EAG/g (Singh et al., 2016). La actividad antioxidante ABTS también fue mayor en la HYC, y estuvo dentro del rango de 23 - $136 \mu \mathrm{mol}$ TE/g encontrado en 35 muestras de yacón procedentes de Perú (Campos et al., 2012).

\section{Análisis colorimétrico de los snacks}

Con el objeto de mejorar las propiedades funcionales de snacks de plátano, se prepararon 4 formulaciones con concentración crecientes de HYC siguiendo la Tabla 1. Los productos obtenidos presentaron el aspecto que se muestra en la Figura 2. 


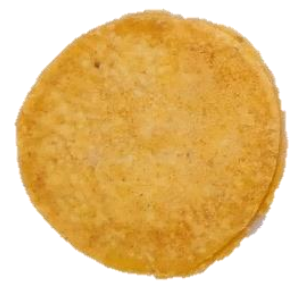

Y0

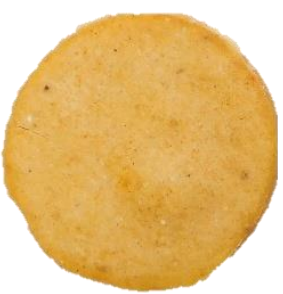

Y7

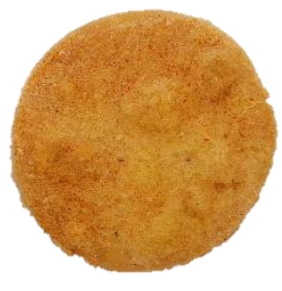

Y13

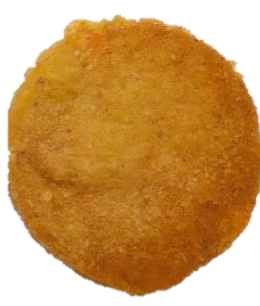

Y20

Fig. 2. Efecto de la adición de harina de yacón en el aspecto visual de los snacks. Códigos según Tabla 1.

Siendo el color uno de los aspectos importantes en la aceptabilidad por el consumidor, se evaluaron los parámetros colorimétricos de los snacks elaborados, los cuales se resumen en la Tabla 3 . Se observó que el parámetro que presentó las mayores variaciones al adicionar la HYC fue la luminosidad $L^{*}$, la cual fue disminuyendo con la mayor incorporación de yacón $(p<0.05)$. Adicionalmente se observó que al incrementar el contenido de HYC, el color viró a tonos amarillos y rojizos. Lo cual pudo ser debido al aporte de pigmentos propios del yacón o por compuestos coloreados formados durante el proceso de horneo. En el horneo por microondas, se podría descartar los cambios de color por las reacciones de pardeamiento no enzimático, ya que el calor es absorbido por la muestra y el aire alrededor de la muestra es frío donde la temperatura de la superficie del producto no puede alcanzar los niveles altos requeridos para que ocurra las reacciones (Içöz, Sumnu y Sahin, 2004). En la Tabla 3 los resultados se expresan como la media \pm desviación estándar media, $(n=3)$. Superíndices diferentes en la misma fila son significativamente diferentes $(p<0.05)$.

Tabla 3: Patrón colorimétrico de los snacks de plátano y yacón, códigos de formulación según Tabla 1.

\begin{tabular}{|c|c|c|c|c|}
\hline Coordenadas de color & $Y 0$ & $Y 7$ & $Y 13$ & $Y 20$ \\
\hline $\mathrm{L}^{*}$ & $56.76 \pm 1.16^{\mathrm{a}}$ & $59.06 \pm 1.10^{\mathrm{b}}$ & $57.59 \pm 0.53^{\mathrm{ab}}$ & $52.11 \pm 0.77^{\mathrm{c}}$ \\
\hline $\mathrm{a}^{*}$ & $9.40 \pm 0.31^{\mathrm{a}}$ & $10.04 \pm 1.04^{\mathrm{a}}$ & $11.17 \pm 0.24^{\mathrm{b}}$ & $10.24 \pm 0.36^{\mathrm{ab}}$ \\
\hline $\mathrm{b}^{*}$ & $38.12 \pm 0.59^{\mathrm{a}}$ & $41.70 \pm 1.46^{\mathrm{b}}$ & $40.09 \pm 0.45^{\mathrm{ab}}$ & $39.37 \pm 0.04^{\mathrm{b}}$ \\
\hline $\mathrm{C}^{*}$ & $39.26 \pm 0.54^{\mathrm{a}}$ & $42.90 \pm 1.66^{\mathrm{b}}$ & $41.61 \pm 0.50^{\mathrm{bc}}$ & $40.68 \pm 0.11^{\mathrm{ac}}$ \\
\hline $\mathrm{h}^{*}$ & $76.14 \pm 0.01^{\mathrm{ab}}$ & $76.48 \pm 0.02^{\mathrm{b}}$ & $74.43 \pm 0.00^{\mathrm{a}}$ & $75.42 \pm 0.01^{\mathrm{c}}$ \\
\hline
\end{tabular}

Análisis de contenido de polifenoles totales y actividad antioxidantes ABTS de los snacks

EI CPT y la actividad antioxidante ABTS en la formulación YO (Tabla 4) fueron inferiores a los valores encontrados en la HPT (Tabla 2), dado que la composición de Y0 fue básicamente HPT, este dato sugiere que se presentaron pérdidas de compuestos fenólicos durante el tratamiento térmico con microondas, lo cual ha sido reportado previamente en otras matrices alimentarias (Jaiswal et al., 2013; Zielinska et al., 2016). La adición creciente de HYC incrementó el CPT y la actividad antioxidante ABTS en los snacks, tal como se esperaba, con un $(\mathrm{P}<0.05)$. Los resultados mostraron la efectividad de fortificar los snacks con HYC para incrementar su funcionalidad con agentes antioxidantes, los cuales están relacionados con la prevención de enfermedades degenerativas.

EI CPT de los snacks del presente estudio fue superior a la de algunas frutas como el borojó (0.418 mg EAG /g), cupuazú (0.403 mg EAG / g), granadilla gigante $(0.707 \mathrm{mg} \mathrm{EAG} \mathrm{/} \mathrm{g),} \mathrm{tomate} \mathrm{melocotón} \mathrm{(0.709} \mathrm{mg} \mathrm{EAG}$ / g) y nuez de macadamia (0.387 mg EAG /g) (Contreras-Calderón et al., 2011). Las formulaciones también presentaron valores superiores a un snacks formulados con arroz, sémola de trigo y afrecho de la cerveza (0.008 mg EAG /g) (Reis y Abu-Ghannam, 2014). En la Tabla 4 los resultados se expresan como la media \pm desviación estándar media $(n=3)$. Superíndices diferentes en la misma columna son significativamente diferentes $(p<0.05)$.

Tabla 4: Fenoles totales y actividad antioxidante ABTS de snacks de plátano y yacón, códigos de formulación según Tabla 1.

\begin{tabular}{|c|c|c|}
\hline Formulación & $C P T[m g E A G / g]$ & $A B T S[\mu m o l E T / g]$ \\
\hline Y0 & $1.57 \pm 0.08^{\mathrm{a}}$ & $2.41 \pm 0.55^{\mathrm{a}}$ \\
\hline $\mathrm{Y} 7$ & $2.39 \pm 0.04^{\mathrm{b}}$ & $3.93 \pm 0.82^{\mathrm{b}}$ \\
\hline $\mathrm{Y} 13$ & $3.93 \pm 0.28^{\mathrm{c}}$ & $4.99 \pm 0.63^{\mathrm{bc}}$ \\
\hline $\mathrm{Y} 20$ & $8.62 \pm 0.14^{\mathrm{d}}$ & $5.24 \pm 0.50^{\mathrm{c}}$ \\
\hline
\end{tabular}




\section{Digestibilidad in vitro del almidón e índice glucémico estimado de los snacks}

Los resultados de la digestibilidad in vitro de las formulaciones de plátano y yacón se presentan en la Tabla 5 , donde se muestra el \% de AT y sus fracciones ARD, ALD y AR en base seca. El contenido de AT para las diferentes formulaciones fue menor al del pan blanco, lo que indicó la presencia de carbohidratos no amiláceos en las formulaciones. El contenido de ARD en los snacks varió entre $34.69 \%$ y $31.14 \%$, valores significativamente menores respecto al pan blanco $(p<0.05)$, así como con respecto a otros productos de baja humedad como snacks de harina de plátano combinado con harinas de maíz y garbanzo $(45.7 \%$ a $48.4 \%)$ (Flores-Silva et al., 2015) y barras de cereal con plátano (51.9) (Utrilla-Coello et al., 2011). Un bajo contenido de ARD es una característica deseable, ya que se relaciona con una baja liberación de glucosa después de la ingesta del alimento.

Todas las formulaciones que contenían HYC mostraron fracciones de ALD y AR mayores que la formulación con solo plátano, este resultado puede atribuirse al contenido de fructooligosacáridos del yacón, considerados como carbohidratos no digeribles ((Valdez Clinis et al., 2013). Productos que contengan altos valores de ALD y AR se consideran beneficiosos, porque estas fracciones de almidón se han relacionado con la capacidad de promover la saciedad, minimizar la secreción de insulina postprandial y minimizar el riesgo de enfermedades cardiovasculares y diabetes (García-Solís et al., 2018). En la Tabla 5 los resultados son la media \pm desviación estándar media $(n=3)$. Superíndices diferente en la misma columna son significativamente diferentes $(p<0.05)$.

Tabla 5: Parámetros de digestibilidad del almidón e índice glucémico estimado de los snacks.

\begin{tabular}{|c|c|c|c|c|c|c|}
\hline Formulación & $A R D$ & $A L D$ & $A R$ & $A T$ & $I H, \%$ & $e I G, \%$ \\
\hline Y0 & $34.69 \pm 1.20^{\mathrm{a}}$ & $6.39 \pm 1.20^{\mathrm{a}}$ & $0.02 \pm 0.01^{\mathrm{a}}$ & 41.10 & $49.37 \pm 1.02^{\mathrm{a}}$ & $50.39 \pm 0.71^{\mathrm{a}}$ \\
\hline Y7 & $31.14 \pm 1.07^{\mathrm{b}}$ & $14.67 \pm 1.07^{\mathrm{b}}$ & $0.36 \pm 0.03^{\mathrm{b}}$ & 46.17 & $51.96 \pm 1.00^{\mathrm{b}}$ & $52.30 \pm 1.13^{\mathrm{bc}}$ \\
\hline Y13 & $34.86 \pm 2.55^{\mathrm{ab}}$ & $10.29 \pm 2.55^{\mathrm{c}}$ & $0.04 \pm 0.01^{\mathrm{a}}$ & 45.19 & $53.03 \pm 1.00^{\mathrm{b}}$ & $53.99 \pm 1.00^{\mathrm{b}}$ \\
\hline Y20 & $31.80 \pm 1.58^{\mathrm{a}}$ & $12.01 \pm 1.58^{\mathrm{bc}}$ & $0.30 \pm 0.01^{\mathrm{c}}$ & 44.10 & $49.93 \pm 1.01^{\mathrm{a}}$ & $51.04 \pm 1.00^{\mathrm{ac}}$ \\
\hline Pan Blanco & $52.09 \pm 1.63^{\mathrm{d}}$ & $43.68 \pm 2.30^{\mathrm{d}}$ & $0.37 \pm 0.04^{\mathrm{d}}$ & 96.14 & $100.00 \pm 0.00^{\mathrm{d}}$ & $94.40 \pm 0.00^{\mathrm{d}}$ \\
\hline
\end{tabular}

Las cuatro formulaciones presentaron un área bajo la curva de hidrólisis más baja que el pan blanco usado como referencia, indicando la presencia de un alto contenido de carbohidratos indigeribles en las cuatro formulaciones estudiadas (Figura 3), y que explica los valores bajos de los índices IH y elG (Tabla 5). Los resultados indicaron que los snacks se pueden clasificar como productos de bajo elG. A pesar de que los valores del elG entre las formulaciones presentaron diferencias significativas $(p<0.05)$, se observó un leve aumento cuando se agregó la HYC, sugiriendo que la HPT fue la responsable de los bajos índices. Los bajos elG encontrados, además de la naturaleza de las harinas usadas, podrían también derivar del bajo contenido de agua en la matriz preparada, que limitaría el grado de gelatinización de los gránulos de almidón presente en la HPT, es reconocido que la parcial gelatinización de los gránulos de almidón favorece una baja digestibilidad del almidón (Miao et al., 2015). Los resultados encontrados en el presente estudio son relevantes porque según Food and Agricutural Organization. (2001), alimentos que presenten resultados de bajo elG son recomendados para personas que posean enfermedades no transmisibles como: diabetes, obesidad y sobrepeso.

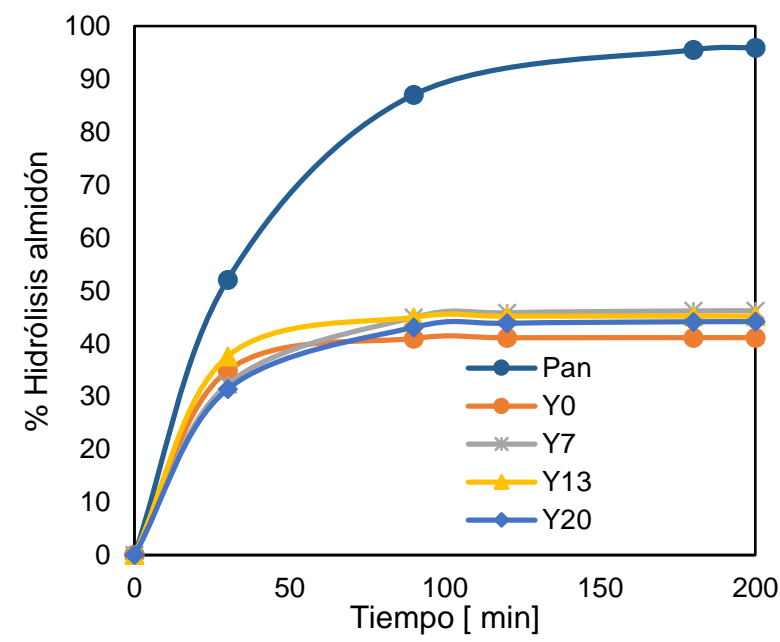

Fig. 3: Cinéticas de hidrólisis del almidón de pan blanco (como referencia) y de las formulaciones. Código de las formulaciones según Tabla 1. 


\section{Análisis sensorial}

La prueba de ordenamiento preferencial (Friedman) obtuvo un $F_{\text {Prueba }}=18.87$ y un $F_{\text {valor }}$ Crítico $=5.99$, considerando el número de evaluadores (30), el número de productos (3) y el riesgo escogido $(\alpha<0.05)$, la Ho es rechazada, indicando que existen diferencias significativas entre las muestras. Existen diferencias consistentes entre las órdenes jerárquicas de los productos. De la prueba de ordenamiento se obtuvo que la muestra preferida por los participantes fue la formulación con adición de 13\% de HYC (Y13) y la que menos prefirieron fue la formulación con adición de $20 \%$ de HYC, (Figura 4). Los jueces resaltaron un mal sabor en la muestra Y7 y una textura pobre en la Y20.

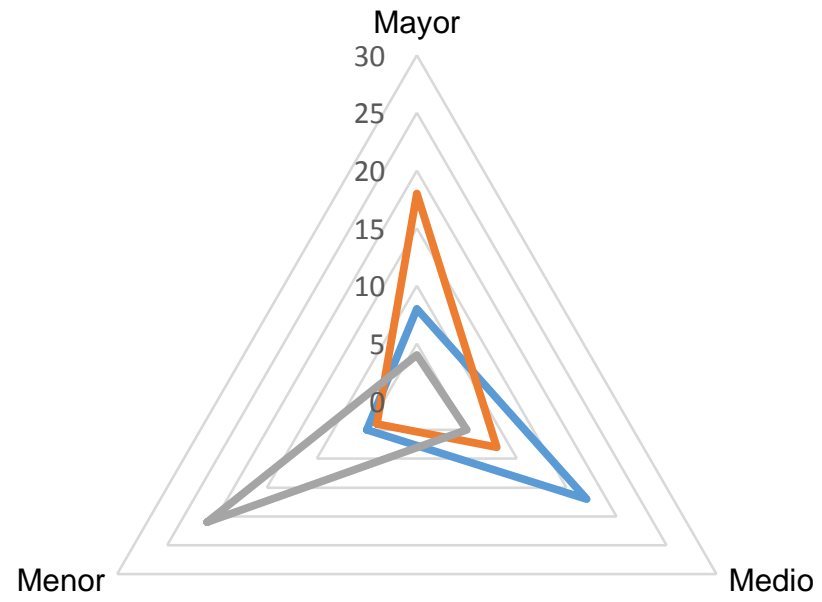

Fig. 4: Preferencia de los evaluadores en los snacks formulados. - Y7, - Y13 y $=$ Y20

\section{CONCLUSIONES}

Las harinas de plátano (HPT) y yacón (HYC) presentaron contenido de polifenoles y actividad antioxidante, siendo la HYC la que presentó mayores valores en estas propiedades. Se observó una reducción del CPT y actividad antioxidante durante el proceso de preparación de los snacks con respecto a los valores obtenidos en las harinas. La creciente incorporación de HYC en las formulaciones de snacks incrementó el CPT y la actividad antioxidante y aportó tonalidades que van desde el amarillo hasta el rojo. En cuanto al elG se encontró que las cuatro formulaciones preparadas se pueden clasificar como alimentos de bajo elG, lo cual se atribuye a la HPT; el incremento de la HYC aumentó ligeramente la fracción de los ALD y AR. El snack de mayor preferencia por los evaluadores sensoriales fue la formulación con $13 \%$ de HYC y la de menor aceptabilidad fue la de $20 \%$ de HYC.

\section{AGRADECIMIENTOS}

Los autores agradecen a la Universidad de Antioquia y al Departamento Administrativo de Ciencia, Tecnología e Innovación - Colciencias por la financiación del proyecto 111566441935.

\section{REFERENCIAS}

Agama-Acevedo, E., J.J. Islas-Hernández y otros tres autores, Starch Digestibility and Glycemic Index of Cookies Partially Substituted with Unripe Banana Flour, doi: 10.1016/j.Iwt.2011.10.010, LWT - Food Science and Technology, 46(1), 177$182(2012)$

Allen, J.C., A.D. Corbitt y otros dos autores, Glycemic Index of Sweet Potato as Affected by Cooking Methods, doi: 10.2174/1874288201206010001, The Open Nutrition Journal, 6(1), 1-11 (2012)

Alvis-Bermudez, A., C. García-Mogollon y S. Dussán-Sarria, Cambios en la Textura y Color en Mango (Tommy Atkins) Pre- Secado por Deshidratación Osmótica y Microondas, doi: 10.4067/S0718-07642016000200005, Información Tecnológica, 27(2), 31-38 (2016)

Anyasi, T. A., A.I. Jideani y G.R. Mchau, Phenolics and Essential Mineral Profile of Organic Acid Pretreated Unripe Banana Flour, doi: 10.1016/j.foodres.2017.09.063, Food Research International, 104, 100-109 (2018)

Augustin, L.S., C.W. Kendall y otros diecisiete autores, Glycemic index, glycemic load and glycemic response: An International Scientific Consensus Summit from the International Carbohydrate Quality Consortium (ICQC), doi: 10.1016/j.numecd.2015.05.005, Nutrition, Metabolism and Cardiovascular Diseases, 25(9), 795-815 (2015)

Benítez-Franco, J., A. Ayala-Aponte y C. Vargas-Serna, Cambios de Color de Yacón (Smallanthus sonchifolius) durante el Secado por Ventana de Refractancia, doi: 10.15446/agron.colomb.v34n1supl.58197, Agronomía Colombiana, 34(September 2017), 994-997 (2016) 
Campos, D., I. Betalleluz-Pallardel y otros cuatro autores, Prebiotic Effects of Yacon (Smallanthus sonchifolius Poepp. \& Endl), a Source of Fructooligosaccharides and Phenolic Compounds with Antioxidant Activity, doi: 10.1016/j.foodchem.2012.05.088, Food Chemistry, 135(3), 1592-1599 (2012)

Castellanos-Galeano, F.J., M. Chavez-Salazar y L. J. Martinez-Hernandez, Effect of Process Variables in the Production of Fried Green Plantain in Vacuum, doi: 10.17533/udea.vitae.v24n1a05, Revista Vitae, 38-46 (2017)

Castro, A., M. Caballero y otros dos autores, Antioxidants in Yacon Products and Effect of Long Term Storage, doi: 10.1590/S0101-20612012005000064, Food Science and Technology, 32(3), 432-435 (2012)

Contreras-Calderón, J., D. Mejía-Díaz y otros seis autores, Evaluation of Antioxidant Capacity in Coffees Marketed in Colombia: Relationship with the Extent of Non-Enzymatic Browning, doi: 10.1016/j.foodchem.2016.04.038, Food Chemistry, 209, 162-170 (2016)

Contreras-Calderón, J., L. Calderón-Jaimes y otros dos autores, Antioxidant Capacity, Phenolic Content and Vitamin C in Pulp, Peel and Seed from 24 Exotic Fruits from Colombia, doi: 10.1016/j.foodres.2010.11.003, Food Research International, 44(7), 2047-2053 (2011)

Flores-Silva, P. C., S. L. Rodriguez-Ambriz. y L. A. Bello-Pérez, Gluten-Free Snacks Using Plantain-Chickpea and Maize Blend: Chemical Composition, Starch Digestibility, and Predicted Glycemic Index, doi: 10.1111/1750-3841.12865, Journal of Food Science, 80(5), C961-C966 (2015)

Food and Agriculture Organization (FAO), World Health Organiztation (WHO), United Nations University (ONU), Human energy requirements: Report of a Joint FAO/WHO/UNU Expert Consultation, Food and Nutrition Technical Report Series, 96. http://doi.org/92 51052123 (2001)

García Soidán, F. J., Riesgo Glucémico y Contribución de la Glucemia Posprandial a la Hemoglobina Glucosilada (HbA1c), doi: 10.1157/13044286, Atencion Primaria, 191-193 (2003)

García-Solís, S. E., L.A. Bello-Pérez y otros dos autores, Plantain flour: A Potential Nutraceutical Ingredient to Increase Fiber and Reduce Starch Digestibility of Gluten-Free Cookies, doi: 10.1002/star.201700107, Starch/Staerke, 70(1-2) (2018)

Goñi, I., A. Garcia-Alonso y F. Saura-Calixto, A starch hydrolysis procedure to estimate glycemic index, doi: 10.1016/S0271-5317(97)00010-9, Nutrition Research, 17(3), 427-437 (1997)

Grandfeldt, Y., I. Björck y otros dos autores, An in Vitro Procedure Based on Chewing to Predict Metabolic Response to Starch in Cereal and Legume Products, European Journal of Clinical Nutrition, 46, 649-660 (1992)

Içöz, D., G. Sumnu y S. Sahin, Color and Texture Development during Microwave and Conventional Baking of Breads, doi: 10.1081/JFP-120025396, International Journal of Food Properties, 7(2), 201-213 (2004)

Ikegwu, O.J., P. A. Okorie y otros dos autores, Effects of Processing Conditions on the Quality of Vacuum Fried Plantain Chips, doi: doi:10.1166/asl.2018.11430, Advanced Science Letters, 24(5), 3527-3530 (2018)

ISO 8587 Sensory analysis - Methodology - Ranking, International Organization for Standardization (2006)

Jaiswal, A. K. y N. Abu-Ghannam, Degradation Kinetic Modelling of Color, Texture, Polyphenols and Antioxidant Capacity of York Cabbage after Microwave Processing, doi: 10.1016/j.foodres.2013.04.007, Food Research International, 53(1), 125-133 (2013)

Khajehei, F., N. Merkt y otros dos autores, Yacon (smallanthus sonchifolius poepp. And endl.) as a Novel Source of Health Promoting Compounds: Antioxidant Activity, Phytochemicals and Sugar Content in Flesh, Peel, and Whole Tubers of Seven Cultivars, doi: 10.3390/molecules23020278, Molecules, 23(2) 278 (2018)

Ludwig, D. S., The Glycemic Index: Physiological Mechanisms Relating to Obesity, Diabetes, and Cardiovascular Disease., doi: 10.1001/jama.287.18.2414, JAMA, 287(18), 2414-23 (2002)

Magallanes-Cruz, P. A., P. C. Flores-Silva y L. A Bello-Perez, Starch Structure Influences its Digestibility: A review. Journal of food science, 82(9), 2016-2023 (2017)

Miao, M., Jiang, B. y otros tres autores, Slowly Digestible Starch-A Review, Critical Reviews in Food Science and Nutrition, 55(12), 1642-1657 (2015)

Parada, J.A. y J. Rozowski, Relación entre la Respuesta Glicémica del Almidón y su Estado Microestructural, doi: 10.4067/S0717-75182008000200001, Revista Chilena de Nutrición, 35(2), 84-92 (2008)

Queiroz, C., A. J. da Silva y otros tres autores, Polyphenol Oxidase Activity, Phenolic Acid Composition and Browning in Cashew Apple (Anacardium occidentale, L.) after Processing, doi: 10.1016/j.foodchem.2010.08.048, Food Chemistry, 125(1), 128-132 (2011)

Reis, S. F. y N. Abu-Ghannam, Antioxidant Capacity, Arabinoxylans Content and In Vitro Glycaemic Index of Cereal-Based Snacks Incorporated with Brewer's Spent Grain, doi: 10.1016/j.Iwt.2013.09.004, LWT - Food Science and Technology, 55(1), 269-277 (2014)

Salinas, J. G, J. A Alvarado y otros dos autores, The Influence of Convection Drying on the Physicochemical Properties of Yacón (Smallanthus sonchifolius), Heat and Mass Transfer, 54(10), 2951-2961 (2018)

Schulze, B., E. M. Hubbermann y K. Schwarz, Stability of Quercetin Derivatives in Vacuum Impregnated Apple Slices after Drying (Microwave Vacuum Drying, Air Drying, Freeze Drying) and Storage, doi: 10.1016/j.lwt.2013.11.021, LWT - Food Science and Technology, 57(1), 426-433 (2014) 
Shamla, L. y P. Nisha, Acrylamide Formation in Plantain (Musa paradisiaca) Chips Influenced by Different Ripening Stages: A Correlation Study with Respect to Reducing Sugars, Amino Acids and Phenolic Content, doi: 10.1016/j.foodchem.2016.12.007, Food Chemistry, 222, 53-60 (2017)

Shodehinde, S. A., A. O. Ademiluyi y otros dos autores, Contribution of Musa Paradisiaca in the Inhibition of $\alpha$-amylase, a-glucosidase and Angiotensin-I converting Enzyme in Streptozotocin Induced Rats, doi: 10.1016/j.Ifs.2015.03.026, Life Sciences, 133, 8-14 (2015)

Singh, B., J.P. Singh y otros dos autores, Bioactive Compounds in Banana and their Associated Health Benefits - A Review, doi: 10.1016/j.foodchem.2016.03.033, Food Chemistry, 206(1 September), 1-11 (2016)

Sousa, S., J. Pinto y otros cinco autores, In Vitro Evaluation of Yacon (Smallanthus sonchifolius) Tuber Flour Prebiotic Potential, doi: 10.1016/j.fbp.2015.04.003, Food and Bioproducts Processing, 95, 96-105 (2015)

Utrilla-Coello, R. G., E. Agama-Acevedo y otros tres autores, Composition and Starch Digestibility of Whole Grain Bars Containing Maize or Unripe Banana Flours, doi: 10.1002/star.201000079, Starch/Staerke, 63(7), 416-423 (2011)

Valdez Clinis, G. A., M. I. Margalef y M. H. Gómez, Formulación de Barra Dietética Funcional Prebiótica a partir de Harina de Yacón (Smallanthus sonchifolius), Diaeta, 31(142), 27-33 (2013)

Van Kleef, E., K. Otten y H. C. Van Trijp, Healthy Snacks at the Checkout Counter: A Lab and Field Study on the Impact of Shelf Arrangement and Assortment Structure on Consumer Choices, doi: 10.1186/1471-2458-12-1072, BMC Public Health, 12(1) 1072 (2012)

Zielinska, M. y A. Michalska, Microwave-assisted Drying of Blueberry (Vaccinium corymbosum L.) Fruits: Drying Kinetics, Polyphenols, Anthocyanins, Antioxidant Capacity, Colour and Texture, doi: 10.1016/j.foodchem.2016.06.003, Food Chemistry, 212, 671-680 (2016) 